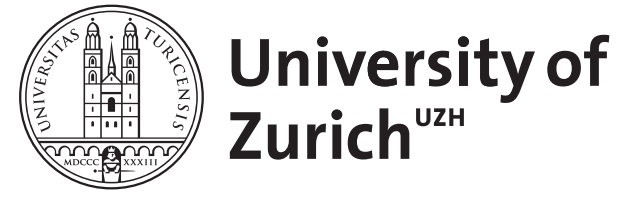

\title{
Simulationen komplexer MR-Anregungsvorgänge
}

Prüssmann, K P ; Crelier, G R ; Boesiger, P

DOI: https://doi.org/10.1515/bmte.1996.41.s1.624

Posted at the Zurich Open Repository and Archive, University of Zurich ZORA URL: https://doi.org/10.5167/uzh-155383

Journal Article

Published Version

Originally published at:

Prüssmann, K P; Crelier, G R; Boesiger, P (1996). Simulationen komplexer MR-Anregungsvorgänge. Biomedizinische Technik. Biomedical engineering, 41(s1):624-625.

DOI: https://doi.org/10.1515/bmte.1996.41.s1.624 


\title{
Simulationen komplexer MR-Anregungsvorgänge
}

\author{
Prüssmann K.P., Crelier G.R., Boesiger P. \\ Institut für Biomedizinische Technik und Medizinische Informatik \\ Universität und ETH Zürich
}

\section{EINLEITUNG :}

Die Wirkung von Magnetresonanz-Anregungspulsen auf die Magnetisierung eines Untersuchungsobjekts kann mit analytischen Methoden im allgemeinen nicht genügend präzise berechnet werden. Dies gilt insbesondere, wenn parallel zur RF-Einstrahlung zeitlich veränderliche Gradienten wirken, wie zum Beispiel bei mehrdimensionalselektiver Anregung, oder wenn bei großer Pulsdauer Relaxationseffekte zu berücksichtigen sind. Zudem können Bewegungen des zu untersuchenden Gewebes, etwa Blutfluß oder die Herzaktion, lange Pulse oder mehrteilige Anregungssequenzen erheblich beeinflussen.

In solchen Fällen kann die zeitliche und räumliche Entwicklung der Magnetisierung durch numerische Rechnungen studiert und optimiert werden. Ausgehend von einer Modellvorstellung der maßgeblichen Effekte sind Methoden zur Lösung der resultierenden Gleichungen erarbeitet worden. Die Lösungsalgorithmen wurden mit einer Bedienungsoberfläche $\mathrm{zu}$ einer komfortablen Simulationssoftware zusammengefasst.

\section{MODELL UND METHODEN :}

Das magnetische Moment eines Gewebevolumens wird als Summe der Momente von Ensembles beschrieben, die gleich zu behandelnde Kernspins zusammenfassen :

$$
\bar{\mu}_{v}(t)=\iiint \bar{M}(\bar{r}, \gamma, \Delta, t) d \bar{r} d \gamma d \Delta
$$

Dabei ist $\mathbf{r}$ der Ort und $\boldsymbol{\gamma}$ der gyromagnetische Faktor des jeweiligen Ensembles. Durch den $\mathrm{B}_{0}$-Offset $\Delta$ werden mikroskopische Feldinhomogenitäten berücksichtigt, die fur Spin-Echos verantwortlich sind. Die zeitliche Entwicklung der so verallgemeinerten Magnetisierung wird nun für jeden Satz von $\{r, \gamma, \Delta\}$ durch eine unabhängige Bloch'sche Gleichung (BG) beschrieben :

$$
\frac{d}{d t}\left(\begin{array}{l}
M_{x} \\
M_{y} \\
M_{z}
\end{array}\right)=\left(\begin{array}{ccc}
-T_{2}^{-1} & \gamma B_{z} & -\gamma B_{y} \\
-\gamma B_{z} & -T_{2}^{-1} & \gamma B_{x} \\
\gamma B_{y} & -\gamma B_{x} & -T_{1}^{-1}
\end{array}\right)\left(\begin{array}{c}
M_{x} \\
M_{y} \\
M_{z}
\end{array}\right)+\left(\begin{array}{c}
0 \\
0 \\
T_{1}^{-1} M_{0}
\end{array}\right)
$$

Das zeitabhängige Magnetfeld $\mathbf{B}(\mathbf{t})$ setzt sich dabei aus dem homogenen Feld $B_{0}$, den Gradientenfeldern $\mathbf{G}(t)$ und der Inhomogenität $\Delta$, sowie der magnetischen Komponente der eingestrahlten Radiowellen zusammen :

$$
\bar{B}(\bar{r}, t)=\left(\begin{array}{c}
B_{1}(t) \cos (\omega t+\varphi(t)) \\
B_{1}(t) \sin (\omega t+\varphi(t)) \\
B_{0}+\bar{r} \cdot \bar{G}(t)+\Delta
\end{array}\right)
$$

$T_{1}(\mathbf{r})$ und $T_{2}(\mathbf{r})$ sind Relaxationszeiten, $M_{0}(r, \gamma, \Delta)$ ist die verallgemeinerte Ruhemagnetisierung. Diese Parameter beschreiben die $\mathrm{zu}$ simulierenden Gewebe und ihre räumliche Anordnung.

Durch ein Geschwindigkeitsfeld $\mathbf{v}(\mathbf{r}, \mathrm{t})$ können beliebige Bewegungen im Untersuchungsobjekt vorgegeben werden. Mit der Wahl eines gewebefesten Koordinatensystems

$\bar{\Re}(\bar{r}, t)=\bar{r}+\int_{0}^{t} \bar{v}(\vec{\Re}(\bar{r}, \tau)) d \tau$

wird das Auftreten von Flußtermen mit partiellen Ableitungen der Magnetisierung in Gleichung (2) vermieden. Die Gleichungen für verschiedene Orte bleiben auf diese Weise entkoppelt und können unabhängig behandelt werden. Dichteänderungen durch divergente Flüsse sind nach dieser Transformation ebenfalls lokal zu berechnen. Letzteres erübrigt sich jedoch häufig, da fast alle menschlichen Gewebe nahezu inkompressibel sind und die zu simulierenden Flüsse entsprechend divergenzfrei.

Aus der Diskretisierung von Gleichung (1) geht eine Anzahl von Gleichungen vom Typ (2) hervor. Nach Transformation in ein mit $\omega$ rotierendes Bezugssystem werden zu ihrer Lösung im wesentlichen drei Verfahren angewendet :

In Phasen der Anregungssequenz ohne RF-Einstrahlung wird die Matrix in (2) für unbewegte Bereiche zeitunabhängig diagonalisiert und das verbleibende, für alle Orte identische Integrationsproblem durch ein Newton-Cotes-Verfahren siebenter Ordnung gelöst. Während der übrigen Intervalle wird ein Runge-KuttaVerfahren vierter Ordnung verwendet. Es wurde so modifiziert, daß analytische Näherungen zur Verminderung der Rechenzeit berücksichtigt werden können. Je nach Stärke, Richtung und Fluktuation des resultierenden Magnetfelds werden in jedem Schritt verschiedene trigonometrische Funktionen oder Potenzreihen als Näherungen bestimmt. Mit diesen Berechnungen ist ein weiteres Runge-Kutta-Verfahren verschachtelt, das bei Koordinatenwechseln die Integralgleichung (4) löst.

Die drei Verfahren werden durch eine Schrittweitensteuerung kontrolliert, die das Fortschreiten jeweils der lokalen Komplexität der Lösung anpaßt. Hierdurch wird einerseits die vom Benutzer vorgegebende Genauigkeit sichergestellt, andererseits die Rechenzeit gegenüber einer fest gewählten Schrittweite erheblich reduziert.

Alle Algorithmen wurden in $\mathrm{C}+$ implementiert und mit einer X-Bedienungsoberfläche und einer GraphikSchnittstelle zu einem Programmpaket verbunden. 


\section{ANWENDUNGEN :}

Das beschriebene Programm ist zur Simulation verschiedener Anregungsverfahren eingesetzt worden.

Bei eindimensional-selektiven Pulsen ist vor allem die Frage nach der Güte des Schichtprofils interessant. Neben verschiedenen Pulsen aus dem Bereich der Bildgebung wurde unter diesem Aspekt die in der MR-Spektroskopie verwendete Sequenz PRESS untersucht [4]. Weitere Simulationen galten zum Beispiel Bewegungsartefakten bei der STEAM-Präparation und Relaxationseffekten bei extrem kurzen $\mathrm{T}_{2}$-Zeiten, wie sie in menschlichem Gewebe bei gebundenem Wasser auftreten.

Für eine zweidimensional-selektive Anregung sollte die k-Raum-Trajektorie der Selektionsgradienten idealerweise eine Fläche bedecken [1]. Da die Trajektorien realer Pulse jedoch nur einen Ausschnitt dieser Fläche mit endlicher Dichte durchlaufen können, treten Abschneide- und Sampling-Artefakte auf. Zudem haben 2D-Pulse je nach der verwendeten Trajektorie recht komplizierte und nicht geschlossen darstellbare Gradienten- und RF-Verläufe und sind durch große Pulsdauern anfällig für Bewegungen. Simulationen solcher Pulse sind daher besonders interessant. Die folgenden Abbildungen zeigen 2DAnregungen eines homogenen, starren Objekts durch Pulse mit nach [3] optimierten Spiraltrajektorien :

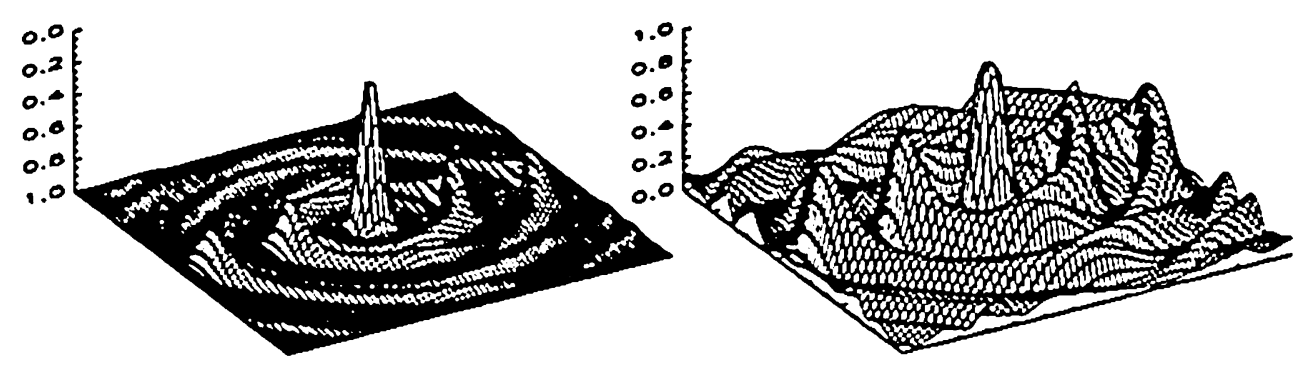

2D-Spiralpuls mit 2 Umdrehungen

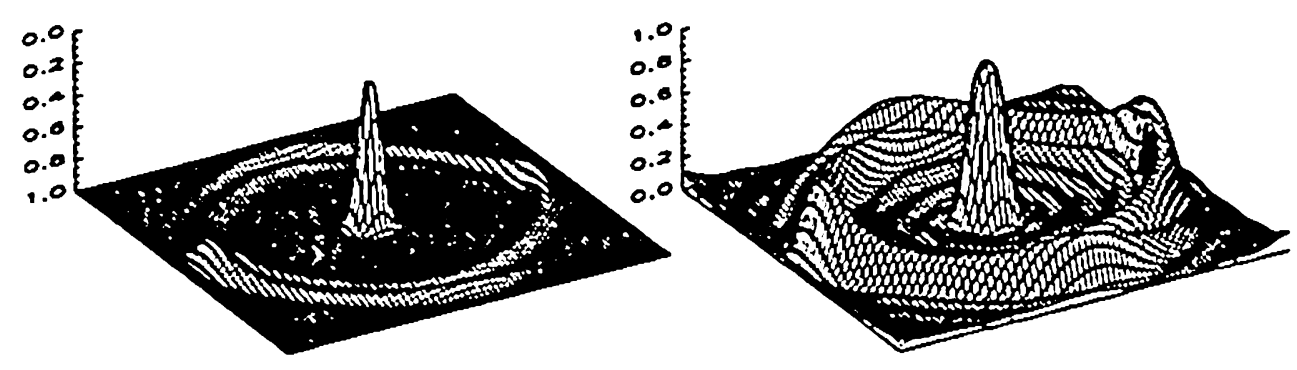

2D-Spiralpuls mit 4 Umdrehungen

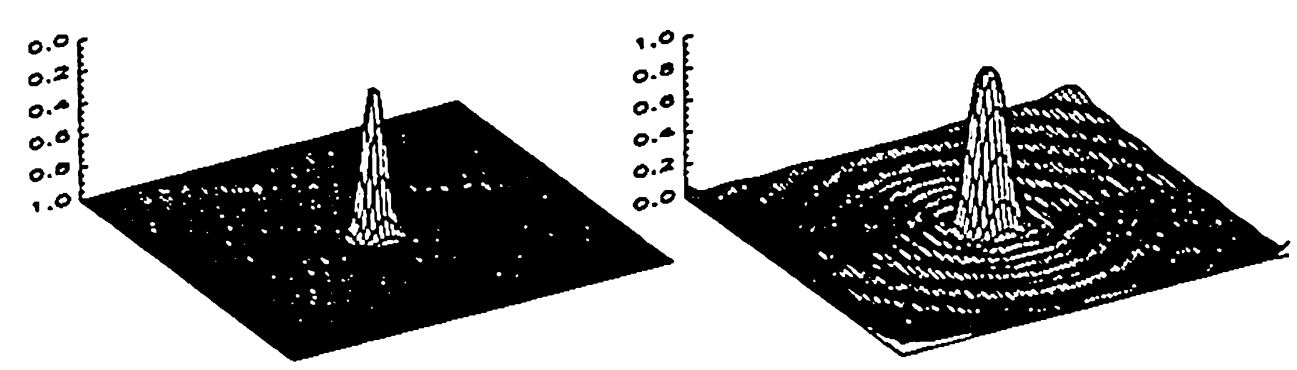

2D-Spiralpuls mit 8 Umdrehungen

Abb.I Longitudinale (links) und transversale Magnetisierung nach der Anregung, dargestellt in einer Ebene senkrecht zur Pulsachse. Die Trajektorien der simulierten Pulse bedecken jeweils das gleiche k-Raum-Fenster mit verschieden vielen Umdrehungen. Der Abstand der ringförmigen Sampling-Artefakte nimmt mit der Dichte der Trajektorie zu. In den untersten Bildern liegt der erste Sampling-Ring außerhalb des dargestellten Bereichs.
Die Beschränkung der k-Raum-Trajektorie auf ein endlich großes Fenster verursacht bei 2D-Anregungen Abschneideartefakte, wie sie bei der transversalen Magnetisierung in Abb.l zu sehen sind. Die Abhängigkeit dieser Störungen von der Größe des überdeckten k-Raum-Fensters illustrieren die folgenden Simulationen :

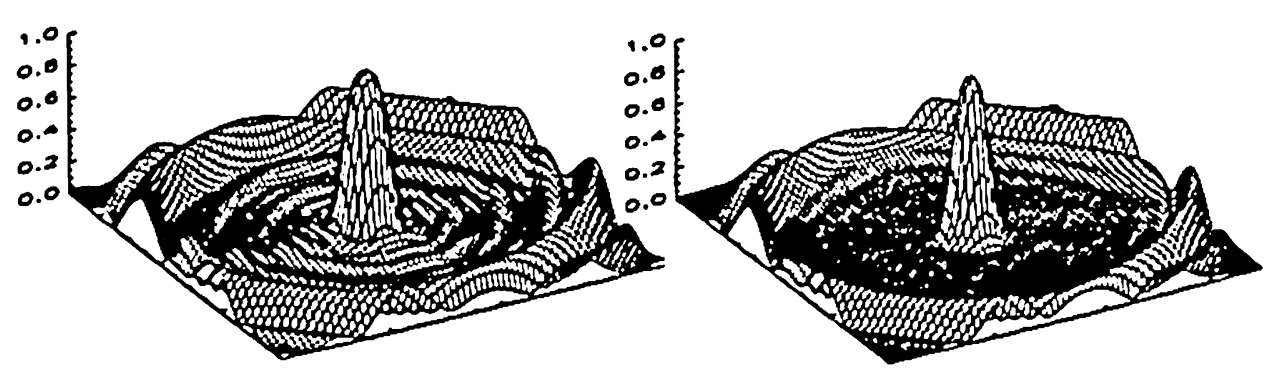

Abb.2 Transversale Magnetisierung nach 2D-Anregung mit Spiraltrajektorien. Links mit vier Umdrehungen in einem $k$-Raum-Fenster der relativen Kantenlänge eins, rechts mit 16 Umdrehungen in einem Fenster der Kantenlänge vier. Die Abschneideartefakte sind rechts entsprechend weniger ausgeprägt, die Sampling-Ringe treten in beiden Fällen in gleichem Abstand auf.

2D-Pulse werden unter anderem zur schnellen Bildgebung am Herzen verwendet [2]. Während der Herzaktion treten an den Herzklappen und in der aufsteigenden Aorta große Geschwindigkeiten auf. Zur Beurteilung möglicher Bewegungsartefakte ist eine 2D-Anregung von schnell fließendem Blut simuliert worden. Die deutlichen Artefakte wurden durch Messungen an einem Flußphantom bestätigt.

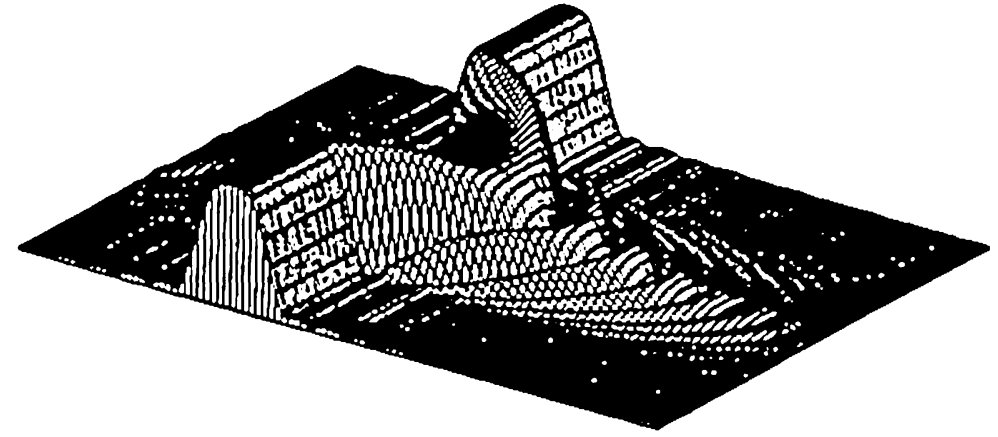

Abb.3 Transversale Magnetisierung nach 2D-Anregung eines Blutgefäßes. Die Pulsachse durchsetzt das $30 \mathrm{~mm}$ starke Gefäß senkrecht und zentral. Die maximale Flußgeschwindigkeit beträgt $1 \mathrm{~m} / \mathrm{s}$, die Pulsdauer $34 \mathrm{~ms}$.

\section{DISKUSSION :}

Mit der vorgestellten Software steht ein Hilfsmittel zur Untersuchung verschiedenster MR-Anregungsvorgänge zur Verfügung. Neben der Berücksichtigung der Effekte von Bewegungen besteht ein wesentlicher Vorzug des Programms in kurzen Rechenzeiten. Sie betragen einige Sekunden für einfache Schichtprofile und bis zu $30 \mathrm{~min}$ für die hier gezeigten Simulationen (DEC AXP 3000).

\section{LITERATUR :}

[1] J.Pauly, D.Nishimura, A.Macovski; J.Magn.Reson. 81,43-56 (1989)

[2] H.E.Cline, C.J. Hardy, J.D.Pearlman; Magn.Reson. Med. 17, 390-401 (1991)

[3] G.R.Crelier, P.Boesiger; Proc.Soc.Magn.Reson.,San Francisco, 119 (1994)

[4] H.Faas et al.; Proceedings BMT 1996 